'Departamento de Nutrición, Diabetes y Metabolismo, Escuela de Medicina, Pontificia Universidad Católica, Santiago, Chile.

${ }^{2}$ Centro de Educación Médica Escuela de Medicina, Pontificia Universidad Católica, Santiago, Chile.

${ }^{3}$ Centro de Nutrición Molecular y Enfermedades Crónicas,

Escuela de Medicina, Pontificia Universidad Católica, Santiago, Chile.

aAlumna de Pregrado, Escuela de Medicina, Pontificia Universidad Católica, Santiago, Chile.

Recibido el 29 de marzo de 2014, aceptado el 15 de agosto de 2014.

Correspondencia a:

Dr. Attilio Rigotti

Departamento de Nutrición, Diabetes y Metabolismo, Escuela de Medicina, Pontificia Universidad Católica de Chile. Marcoleta 367-interior, $2^{\circ}$ Piso, Santiago, Chile.

Teléfono: +56223543862 arigotti@med.puc.cl

\section{Recursos psicosociales positivos y salud cardiovascular}

\author{
MARÍA PÍA NITSCHE ${ }^{1, a}$, MARCELA BITRAN $N^{2,3}$, NURIA PEDRALS $^{3}$, \\ GUADALUPE ECHEVERRÍA ${ }^{3}$, ATTILIO RIGOTTI ${ }^{1,3}$
}

\section{Positive psychosocial factors and cardiovascular health}

Health is defined as a state of complete physical, mental and social wellbeing and not merely as the absence of disease. Thus, medical practice must not only deal with diagnosis and treatment of pathological conditions and solve physical ailments, but also promote a comprehensive wellbeing state -including the psychological domain- to achieve positive health. Therefore, it is necessary to scientifically identify the psychological and social determinants that contribute more effectively to prevent disease and achieve optimal health. This article reviews the most recent evidence showing the importance of positive psychological and social resources on cardiovascular disease, the leading cause of morbidity and mortality as well as health care costs worldwide. Evidence is summarized regarding the role of positive psychosocial factors as health promoters and protectors against cardiovascular risk, the possible mechanisms that explain this association, and the practical implications and future research arising from this perspective. The development of interdisciplinary research in this field, incorporating the area of psychological wellbeing, should help to generate and test new strategies aimed at more effective cardiovascular disease prevention and treatment.

(Rev Med Chile 2014; 142: 1316-1323)

Key words: Cardiovascular diseases; Psychology, medical; Psychosocial factors.
L a OMS define la salud como un estado de completo bienestar físico, mental y social, y no meramente como la ausencia de enfermedades ${ }^{1}$. Por lo tanto, los programas de salud no deben exclusivamente diagnosticar y tratar condiciones patológicas, sino que además deberían fomentar y promover el máximo bienestar integral de las personas.

En este contexto integrativo, los trastornos psicológicos y sociales también son relevantes en el origen y el manejo de las enfermedades físicas. En el área cardiovascular, varios trastornos psicosociales se asocian y predicen el desarrollo y progresión de la enfermedad coronaria ${ }^{2-10}$. Así, la personalidad tipo A, caracterizada por alta competitividad, hostilidad y sensación de urgencia constante, es un factor de riesgo para cardiopatía isquémica, en contraste a lo que ocurre con la personalidad tipo B expresada por menos apremio y agresividad y más paciencia individual. Por lo tanto, se ha propuesto el manejo activo de los aspectos psicosociales negativos que predisponen a enfermedad cardiovascular (ECV) con detección rutinaria de este tipo de factores de riesgo, tratamiento adecuado de pacientes con trastornos psicológicos leves y derivación de casos graves a los especialistas correspondientes.

Como contraparte, la búsqueda de la salud física debe considerar el desarrollo concomitante de recursos psicosociales positivos para alcanzar un bienestar integral ${ }^{1,2,11}$. Desde una perspectiva psicosocial, el bienestar está definido por tres dimensiones teóricamente conectadas, pero empíricamente diferentes: bienestar emocional, 
psicológico y social ${ }^{12}$. La psicología positiva es una nueva especialidad que estudia el bienestar psicosocial junto con sus determinantes individuales y colectivos y desarrolla intervenciones que permitan promoverlo en las personas ${ }^{11,13-18}$. Así, los profesionales de la salud deberían considerar el desarrollo de los recursos psicosociales positivos de sus pacientes para prevenir conductas y factores de riesgo y promover hábitos saludables que eviten las enfermedades y/o faciliten su tratamiento. Este modelo plantea que el bienestar psicológico y social contribuye al funcionamiento óptimo de múltiples sistemas biológicos, lo que ayudaría a mantener un organismo sano, evitando que sucumba a una enfermedad o promoviendo la pronta recuperación de la salud cuando existe una patología.

Este artículo revisa la evidencia que muestra la relevancia de aspectos psicológicos y sociales positivos como elementos promotores de la salud cardiovascular y protectores frente al riesgo de $\mathrm{ECV}$, los eventuales mecanismos que explicarían esta asociación y las implicancias prácticas y estudios a futuro.

\section{Factores psicosociales positivos y salud general}

La sabiduría popular sugiere que los estados psicológicos positivos promueven la salud. Pero, ¿tienen la afectividad positiva, la vitalidad emocional, el optimismo o el bienestar subjetivo un efecto realmente favorable sobre la salud de las personas? Dentro de múltiples estudios, una evidencia llamativa es la relación descrita entre emocionalidad positiva y longevidad ${ }^{19}$ y mayor sobrevida de adultos mayores ${ }^{20}$. Otros trabajos han reportado que el bienestar psicológico y/o las relaciones interpersonales positivas se asocian a mejor respuesta inmune post-vacunación contra infecciones virales ${ }^{21,22}$. Adicionalmente, el optimismo se correlaciona significativa e independientemente con menos biomarcadores intermedios de enfermedad, menor incidencia de patologías y mayor salud física a largo plazo ${ }^{23}$.

\section{Factores psicosociales positivos y menor riesgo de enfermedad cardiovascular}

Un número creciente de estudios prospectivos han relacionado el bienestar y factores psicosociales positivos con indicadores cardiovasculares objetivos como incidencia y recurrencia de enfermedad coronaria y cerebrovascular ${ }^{24}$. Así, el optimismo, el sentido del humor, la religiosidad, la vitalidad, la flexibilidad emocional, la resiliencia y las redes de apoyo social se asocian a menor riesgo de ECV de tipo isquémico ${ }^{6}$. Estas asociaciones son independientes de la presencia de estados psicológicos negativos, indicando que el beneficio cardiovascular determinado por los factores psicosociales positivos se debe a mucho más que la ausencia de enfermedad mental.

En primer lugar, el optimismo -definido como una actitud mental que interpreta positivamente los acontecimientos de la vida- es un recurso psicosocial consistentemente asociado con salud cardiovascular. En décadas previas, se reportó que el optimismo predecía-independientemente de otros factores- mejor recuperación y menor tasa de rehospitalización post-revascularización miocárdica ${ }^{25,26}$. Un estudio de cohorte de adultos mayores en Estados Unidos de Norteamérica describió que la incidencia de enfermedad coronaria era significativamente menor (riesgo relativo de 0,44-0,45 después de un ajuste multivariado) en hombres optimistas versus sujetos pesimistas ${ }^{27}$. El estudio Women's Health Initiative también encontró que la disposición optimista se relaciona con menor mortalidad cardiaca en mujeres postmenopáusicas independiente de otros factores de riesgo cardiovascular ${ }^{28}$. En Holanda, dos estudios independientes han establecido que el optimismo en adultos mayores se asocia a menor riesgo relativo de mortalidad cardiovascular ajustado por otras variables ${ }^{29,30}$. En conjunto, todos estos resultados indican que el optimismo podría proteger contra el riesgo de enfermedad coronaria.

Adicionalmente, un estudio prospectivo en Estados Unidos de Norteamérica -con seguimiento a 15 años plazo- mostró que la presencia de altos niveles basales de vitalidad emocional - definida como sensación de energía emocional positiva- se asociaba con menor riesgo relativo de enfermedad coronaria isquémica fatal y no fatal $(0,81$ (IC: 0,69 $0,94)$ con ajuste multivariado), mostrando una relación tipo dosis-respuesta de una disminución de $2-3 \%$ en el riesgo coronario por cada unidad de aumento en la vitalidad emocional ${ }^{31}$. Adicionalmente, este estudio sugiere que los estilos de vida (actividad física, tabaquismo y consumo de alcohol) tendrían una participación significativa como mediadores de la relación entre vitalidad 
emocional y salud cardiovascular ${ }^{31}$. Además de la vitalidad emocional, la vitalidad mental también se ha correlacionado prospectivamente con reducción del riesgo de varios tipos de eventos cardiovasculares ${ }^{32}$.

La afectividad positiva (experiencia de emociones placenteras como alegría, dicha y felicidad) por sí misma, independiente de la ausencia de depresión, igualmente se asocia a protección cardiovascular. En base a un estudio de seguimiento realizado en Canadá, un modelo de análisis de riesgo proporcional -controlado por edad, género, factores tradicionales de riesgo cardiovascular y emocionalidad negativa- demostró que la afectividad positiva predice un menor riesgo (riesgo relativo 0,78 (IC: 0,63-0,96)) de cardiopatía coronaria isquémica fatal y no fatal ${ }^{33}$. Otro estudio también acaba de asociar la presencia de bienestar positivo con una reducción de $30-50 \%$ en la incidencia de enfermedad coronaria en una población con riesgo cardiovascular, con un mayor efecto en el estrato de más alto riesgo, independiente de los factores predisponentes tradicionales ${ }^{34}$. Como implicancia de estos hallazgos, proponemos que el uso de estrategias que aumenten la afectividad positiva, y no se centren solamente en reducir los síntomas depresivos, podrían tener un importante papel en prevención cardiovascular.

Por otro lado, una adecuada autorregulación emocional también sería beneficiosa para la salud. Así, un estudio prospectivo de cohorte en hombres -sin enfermedad coronaria ni diabetes mellitus al inicio del estudio y seguidos durante un promedio de 13 años- demostró que los individuos con mayores niveles de autorregulación emocional presentaron un menor riesgo ajustado por edad (0,38, IC: $0,22-0,64)$ para outcome combinado de infarto miocárdico no fatal y muerte por cardiopatía coronaria en comparación con los sujetos con menor autorregulación (ajustado por factores de riesgo tradicionales para enfermedad coronaria $y$ afectividad positiva y negativa) ${ }^{35}$.

En 2011, el análisis de la cohorte de Whitehall de $\approx 8.000$ sujetos inicialmente sanos reveló que la satisfacción personal y con la vida, la familia y el trabajo -pero no las actividades de entretenimiento ni el estándar de vida- se asocia a menor riesgo $(-23 \%)$ de ECV isquémica, normalizado por factores demográficos, estilo de vida, niveles de presión arterial y estado metabólico ${ }^{36}$. Más recientemente, el sentido de propósito en la vida de adultos estadounidenses $>50$ años también se correlacionó con menor probabilidad de infarto miocárdico durante un seguimiento a 2 años plazo, incluso controlando por severidad de la enfermedad coronaria, percepción subjetiva de salud y otros factores confundentes ${ }^{37}$.

Actualmente, la promoción de la salud cardiovascular enfatiza el origen temprano de las enfermedades, aunque se conoce poco sobre los factores psicosociales de la infancia que modularían el desarrollo de ECV en la adultez. Recientemente, el análisis de una cohorte de niños del Collaborative Perinatal Project evaluados a los 7 años de edad ha demostrado que una mejor regulación de la atención, mayor capacidad cognitiva y un ambiente positivo en el hogar durante la infancia se asocian en forma aditiva con un estado cardiovascular más favorable en la vida adulta después de un ajuste por variables demográficas y salud cardiovascular $^{38}$. Por lo tanto, la presencia de factores psicosociales positivos en la niñez podría promover una mayor salud cardiovascular en la adultez. Así, la prevención cardiovascular podría considerar intervenciones centradas en el desarrollo de recursos psicosociales durante la infancia. Incluso más, el impacto favorable de una infancia con relaciones intrafamiliares saludables (p.e, una relación madre-hijo positiva) se proyecta a lo largo de toda la vida, alcanzando beneficio en términos de menor riesgo de demencia y otras enfermedades crónicas durante la tercera edad ${ }^{39}$.

\section{Posibles mecanismos explicativos de la relación entre bienestar psicológico y salud cardiovascular}

En base a la evidencia acumulada, la relación inversa entre indicadores de bienestar psicológico positivo y ECV ha ido adquiriendo cada vez mayor fuerza. La pregunta que surge es: ¿cuáles serían los posibles mecanismos que explicarían la relación inversa entre marcadores de bienestar y ECV? El conocimiento actual sobre este tema es escaso y la mayoría de los trabajos corresponden a estudios de tipo transversal y un número menor de cohortes longitudinales.

Analizando el correlato neuroendocrino, cardiovascular e inflamatorio de la afectividad positiva -ajustado por otras variables relevantes-, se ha establecido una relación inversa del bienestar subjetivo con niveles de cortisol salival, variabili- 
dad anormal del ritmo cardiaco y respuesta inflamatoria post-estrés ${ }^{40}$. Igualmente, las personas más proclives a perdonar o con mayor apoyo social muestran menor frecuencia cardiaca y elevaciones más moderadas de la presión arterial frente a estrés ${ }^{41,42}$. Además, el optimismo se asocia con menor inflamación y menor disfunción endotelial medida por los niveles circulantes de interleuquina 6 y la molécula de adhesión intercelular tipo 1, respectivamente ${ }^{43}$. Las respuestas mostradas por estos parámetros en relación al bienestar psicológico se asocian per se a mayor salud cardiovascular y menor riesgo de ateroesclerosis.

Por otro lado, la presencia espontánea o la inducción experimental de bienestar psicológico y emocionalidad positiva se traduce en mejor balance funcional del sistema nervioso autónomo y desactivación del eje hipotálamo-hipófisis-suprarrenal $^{17,24}$. Así, la afectividad positiva aumenta los niveles de acetilcolina, la variabilidad de la frecuencia cardiaca, la recuperación post-estrés y mejora el control de la presión arterial, mientras reduce los niveles de norepinefrina, la frecuencia cardiaca basal y la hiperreactividad cardiovascular frente a estrés, siendo todos estos indicadores de un sistema cardiovascular más saludable y menos propenso a ECV.

La estrecha relación entre bienestar psicosocial y salud cardiovascular también estaría mediada por la práctica de estilos de vida más saludables, incluyendo una mejor alimentación, hábitos de sueño adecuados, mayor actividad física, consumo moderado de alcohol y ausencia de tabaquismo. La asociación entre los recursos psicosociales y una dieta más saludable es muy consistente, sobre todo cuando se analiza el optimismo, aunque los resultados varían dependiendo de qué aspecto de la dieta se considera en cada caso. Así, el optimismo se ha correlacionado con mayor ingesta de frutas y verduras, fibra y productos lácteos bajo en grasas $^{44}$. El sentido de satisfacción con la vida también se ha asociado a un mayor consumo de frutas y menor ingesta de alimentos grasos en estudiantes universitarios de Estados Unidos de Norteamérica, Europa y Asia ${ }^{45}$. Sin embargo, la dirección causal uni o bidireccional de estas asociaciones permanece incierta debido a que casi todos los trabajos son de tipo transversal, requiriéndose estudios prospectivos a futuro.

Los indicadores de bienestar también se asocian a mayor actividad física ${ }^{24}$. En estos estudios, la evaluación de actividad física fue hecha por autorreporte (por lo que podría estar sobreestimada) y el bienestar por mediciones que analizan la emocionalidad positiva y satisfacción con la vida, más que el optimismo y un sentido psicológico más profundo del bienestar. De hecho, el bienestar subjetivo y el optimismo no se correlacionan con mayor actividad física en estudios longitudinales ${ }^{46,47}$, aunque sí ocurre en estudios transversales ${ }^{48-50}$. En contraste, tanto estudios transversales como de cohortes longitudinales han establecido que los individuos más felices o con mayor bienestar hedónico practican más ejercicio ${ }^{24}$. Por lo tanto, el desarrollo de bienestar psicológico puede tener un efecto beneficioso a largo plazo disminuyendo el sedentarismo, pero se necesitan estudios adicionales para demostrarlo más directamente.

Adicionalmente, diferentes indicadores de bienestar -hedónico o eudamónico, optimismo y vitalidad subjetiva- se relacionan inversamente con el tabaquismo en estudios transversales ${ }^{51,52}$. Los estudios longitudinales disponibles también sugieren una asociación entre mayor vitalidad, optimismo y esperanza con un menor riesgo de fumar ${ }^{53}$. Finalmente, algunos estudios transversales indican -aunque no siempre en forma consistente- que el bienestar psicológico y el optimismo parecen asociarse positivamente con una ingesta moderada de alcohol y hábitos de sueño reparadores, e inversamente con abstinencia alcohólica, alcoholismo exagerado o sueño inadecuado ${ }^{24}$.

La presencia de factores psicosociales positivos mejora la adherencia a tratamientos no farmacológicos y farmacológicos, lo cual reduce la necesidad de atención ambulatoria y hospitalización, la morbimortalidad cardiovascular y los costos de salud. Por ejemplo, un meta-análisis reveló que la adherencia al tratamiento médico está fuertemente influenciada por el apoyo social derivado de la relación de pareja, la cohesión familiar y las redes de apoyo ${ }^{54}$. En este contexto, una parte del efecto favorable del apoyo social sobre la adherencia al tratamiento farmacológico se podría explicar por un componente emocional y psicológico positivo que favorecería el autocuidado del paciente.

En resumen, la evidencia -principalmente de estudios transversales- indica que el bienestar y los recursos psicosociales positivos se asocian positivamente con conductas saludables y negativamente con comportamientos nocivos para la salud. 


\section{Perspectivas futuras sobre la relación de los recursos psicológicos positivos con salud y enfermedad cardiovascular}

El surgimiento de esta evidencia de asociación entre factores psicológicos y salud, y de los posibles mecanismos relacionados, se asemeja a lo ocurrido en la segunda mitad del siglo XX cuando aparecieron los estudios de asociación entre los factores de riesgo actualmente aceptados para el desarrollo de ECV como la hipercolesterolemia. Estos trabajos generaron hipótesis de relación causa-efecto que se validaron en estudios de intervención prospectivos y que actualmente sustentan el uso de hipolipemiantes en la prevención y el tratamiento de la ECV.

Actualmente, existe un número creciente de intervenciones conductuales para reducir eventos cardiacos en pacientes con factores de riesgo psicosocial. Aunque la eficacia independiente de estas intervenciones psicosociales no está totalmente establecida -probablemente por la necesidad de grandes tamaños muestrales así como por la longitud del seguimiento y los altos costos requeridos para estos ensayos-, diferentes estrategias de rehabilitación cardiaca multifactorial -que incluyen intervenciones psicosociales- han demostrado una reducción de eventos cardiovasculares ${ }^{55}$.

La evidencia derivada de la psicología positiva indica que los recursos psicosociales positivos se pueden desarrollar y mejorar activamente ${ }^{56} \mathrm{y}$, por lo tanto, permite vislumbrar nuevas oportunidades para el fomento de la salud y la prevención de enfermedades. De hecho, la inducción de afectividad positiva mediante una intervención psicoeducacional controlada y randomizada en pacientes post-revascularización miocárdica determinó un aumento sostenido y clínicamente significativo en actividad física a 12 meses de seguimiento ${ }^{57}$. Una intervención similar -en comparación con un manejo estándar- determinó un mayor cumplimiento del tratamiento farmacológico en pacientes hipertensos ${ }^{58}$. Varios mecanismos explicarían estos hallazgos: la afectividad positiva podría facilitar la conexión que establece el paciente entre su esfuerzo, el cambio de conducta y el sentido de autoeficacia, atenuar los efectos negativos de la depresión sobre la adherencia a la medicación y estimular la adopción de comportamientos de salud más positivos. Estos resultados son consistentes con evidencia previa que indica que las interven- ciones psicoconductuales tienen mayor éxito -en comparación con intervenciones exclusivamente informativas y educacionales- sobre la adherencia al uso de fármacos cardiovasculares ${ }^{59}$. Sin embargo, se requieren estudios más prolongados para establecer si los cambios favorables observados en el estilo de vida y en la adherencia a tratamiento en los grupos intervenidos se traducen realmente en una mejoría de resultados clínicos a largo plazo.

En la actualidad, no se conocen cabalmente los mecanismos que permiten incorporar conductas saludables en los seres humanos. Uno de los modelos más influyentes es la teoría de la autodeterminación, la cual propone que la satisfacción de las necesidades psicológicas básicas permite alcanzar una verdadera motivación intrínseca para lograr un funcionamiento óptimo y el desarrollo del máximo potencial personal, incluyendo la adquisición de cambios de estilo de vida beneficiosos para el cuidado en salud. De hecho, varias intervenciones en el contexto médico basadas en este modelo favorecen la abstinencia prolongada de tabaco, la mantención de pérdida de peso y el aumento de actividad física ${ }^{60,61}$, todos elementos claves para la prevención de la ECV.

\section{Conclusión}

Considerando la definición que propone la OMS, la salud positiva debe definirse empíricamente para establecer científicamente los componentes activos de origen biológico, psicológico y social que permitan prevenir más efectivamente las enfermedades y alcanzar un verdadero estado de salud y bienestar integral del ser humano.

La evidencia revisada sustenta la idea que un funcionamiento psicológico saludable, y no sólo la ausencia de factores psicológicos negativos, puede tener importantes beneficios cardiovasculares. Dado que se puede promover el bienestar psicológico mediante estrategias validadas experimentalmente, estos hallazgos ofrecen nuevas perspectivas para la prevención y el tratamiento de la ECV. Por otro lado, no debemos olvidar el impacto beneficioso adicional que podría tener el desarrollo de recursos psicosociales positivos en los mismos profesionales de la salud (p.e., optimismo, empatía, atención plena) sobre la calidad de la relación con los pacientes y la efectividad de la implementación de medidas preventivas o tratamientos de sus estados patológicos. 
El abordaje futuro de esta temática requerirá de investigación interdisciplinaria que integre teorías y metodologías derivadas de la fisiología, fisiopatología, clínica, neurociencia, epidemiología, psicología y sociología para que se traduzca en un mejor manejo de enfermedades que determinan gran morbimortalidad y costos de salud en el mundo actual. Este avance también deberá reflejarse en la introducción de modificaciones en la docencia clínica que permitan formar futuros profesionales capaces de dar una atención integral a sus pacientes y educarlos en el cultivo de los factores psicosociales positivos para poder ser partícipes del autocuidado en salud y de la promoción de su propio bienestar integral.

\section{Referencias}

1. WHO. Constitution of World Health Organization, New York, 1946. http://apps.who.int/gb/bd/PDF/bd47/EN/ constitution-en.pdf.

2. WHO. International classification of functioning, disability and health: ICF. Geneva, Switzerland. 2001.

3. Yusuf S, Hawken S, Ounpuu S, Dans T, Avezum A, Lanas $F$, et al, INTERHEART Study Investigators. Effect of potentially modifiable risk factors associated with myocardial infarction in 52 countries (the INTERHEART study): case-control study. Lancet 2004; 364: 937-52.

4. Rosengren A, Hawken S, Ounpuu S, Sliwa K, Zubaid $\mathrm{M}$, Almahmeed WA, et al, INTERHEART investigators. Association of psychosocial risk factors with risk of acute myocardial infarction in 11119 cases and 13648 controls from 52 countries (the INTERHEART study): case-control study. Lancet 2004; 364: 953-62.

5. Rozanski A, Blumenthal JA, Davidson KW, Saab PG, Kubzansky L. The epidemiology, pathophysiology, and management of psychosocial risk factors in cardiac practice: the emerging field of behavioral cardiology. J Am Coll Cardiol 2005; 45: 637-51.

6. Thomas RJ. Behavioral cardiology: where the heart and head meet. Business Briefing: US Cardiology 2006: 1-5.

7. Williams RB. Psychosocial and biobehavioral factors and their interplay in coronary heart disease. Annu Rev Clin Psychol 2008; 4: 349-65.

8. Thurston RC, Kubzansky LD. Women, loneliness, and incident coronary heart disease. Psychosom Med 2009; 71: 836-42.

9. Hamer M, Malan L. Psychophysiological risk markers of cardiovascular disease. Neurosci Biobehav Rev 2010; 35: 76-83.
10. Miller G. Why loneliness is hazardous to your health. Science. 2011; 331: 138-40.

11. Ryff CD, Singer BH, Dienberg Love G. Positivehealth: connecting well-being with biology. Philos Trans R Soc Lond B Biol Sci 2004; 359: 1383-94.

12. Keyes CL. The mental health continuum: from languishing to flourishing in life. J Health Soc Behav 2002; 43: 207-22.

13. Seligman ME, Csikszentmihalyi M. Positive psychology. An introduction. Am Psychol 2000; 55: 5-14.

14. Diener E, Seligman ME. Very happy people. Psychol Sci 2002; 13: 81-4.

15. Lyubomirsky S, King L, Diener E. The benefits of frequent positive affect: does happiness lead to success? Psychol Bull 2005; 131: 803-55.

16. Chida Y, Steptoe A. Positive psychological well-being and mortality: a quantitative review of prospective observational studies. Psychosom Med 2008; 70: 741-56.

17. Steptoe A, O’Donnell K, Marmot M, Wardle J. Positive affect and psychosocial processes related to health. Br J Psychol 2008; 99: 211-27.

18. Veenhoven RH. Healthy happiness: effects of happiness on physical health and the consequences for preventive health care. J Happ Studies 2008, 9: 449-69.

19. Danner DD, Snowdon DA, Friesen WV. Positive emotions in early life and longevity: findings from the nun study. J Pers Soc Psychol 2001; 80: 804-13.

20. Steptoe A, Wardle J. Positive affect measured using ecological momentary assessment and survival in older men and women. Proc Natl Acad Sci USA 2011; 108: 18244-8.

21. Hayney MS, Love GD, Buck JM, Ryff CD, Singer B, Muller D. The association between psychosocial factors and vaccine-induced cytokine production. Vaccine 2003; 21 : 2428-32.

22. Rosenkranz MA, Jackson DC, Dalton KM, Dolski I, Ryff $\mathrm{CD}$, Singer $\mathrm{BH}$, et al. Affective style and in vivo immune response: neurobehavioral mechanisms. Proc Natl Acad Sci USA 2003; 100: 11148-52.

23. Rasmussen HN, Scheier MF, Greenhouse JB. Optimism and physical health: a meta-analytic review. Ann Behav Med 2009; 37: 239-56.

24. Boehm JK, Kubzansky LD. The heart's content: the association between positive psychological well-being and cardiovascular health. Psychol Bull 2012; 138: 65591.

25. Scheier MF, Matthews KA, Owens JF, Magovern GJ Sr, Lefebvre RC, Abbott RA, et al. Dispositional optimism and recovery from coronary artery bypass surgery: the beneficial effects on physical and psychological wellbeing. J Pers Soc Psychol 1989; 57: 1024-40.

26. Scheier MF, Matthews KA, Owens JF, Schulz R, Bridges 
MW, Magovern GJ, et al. Optimism and rehospitalization after coronary artery bypass graft surgery. Arch Intern Med 1999; 159: 829-35.

27. Kubzansky LD, Sparrow D, Vokonas P, Kawachi I. Is the glass half empty or half full? A prospective study of optimism and coronary heart disease in the normative aging study. Psychosom Med 2001; 63: 910-6.

28. Tindle HA, Chang YF, Kuller LH, Manson JE, Robinson JG, Rosal MC, et al. Optimism, cynical hostility, and incident coronary heart disease and mortality in the Women's HealthInitiative. Circulation 2009; 120: 656-66.

29. Giltay EJ, Geleijnse JM, Zitman FG, Hoekstra T, Schouten EG. Dispositional optimism and all-cause and cardiovascular mortality in a prospective cohort of elderly Dutch men and women. Arch Gen Psychiatry 2004; 61: 1126-35.

30. Giltay EJ, Kamphuis MH, Kalmijn S, Zitman FG, Kromhout D. Dispositional optimism and the risk of cardiovascular death: the Zutphen Elderly Study. Arch Intern Med 2006; 166: 431-6.

31. Kubzansky LD, Thurston RC. Emotional vitality and incident coronary heart disease: benefits of healthy psychological functioning. Arch Gen Psychiatry 2007; 64: 1393-401.

32. Richman LS, Kubzansky LD, Maselko J, Ackerson LK, Bauer M. The relationship between mental vitality and cardiovascular health. Psychol Health 2009; 24: 919-32.

33. Davidson KW, Mostofsky E, Whang W. Don't worry, be happy: positive affect and reduced 10-year incident coronary heart disease: the Canadian Nova Scotia Health Survey. Eur Heart J 2010; 31: 1065-70.

34. Yanek LR, Kral BG, Moy TF, Vaidya D, Lazo M, Becker LC, et al. Effect of positive well-being on incidence of symptomatic coronary artery disease. Am J Cardiol 2013; 112: 1120-5.

35. Kubzansky LD, Park N, Peterson C, Vokonas P, Sparrow D. Healthy psychological functioning and incident coronary heart disease: the importance of self-regulation. Arch Gen Psychiatry 2011; 68: 400-8.

36. Boehm JK, Peterson C, Kivimaki M, Kubzansky LD. Heart health when life is satisfying: evidence from the Whitehall II cohort study. Eur Heart J 2011; 32: 2672-7.

37. Kim ES, Sun JK, Park N, Kubzansky LD, Peterson C. Purpose in life and reduced risk of myocardial infarction among older U.S. adults with coronary heart disease: a two-year follow-up. J Behav Med 2013; 36: 124-33.

38. Appleton AA, Buka SL, Loucks EB, Rimm EB, Martin LT, Kubzansky LD. A prospective study of positive early-life psychosocial factors and favorable cardiovascular risk in adulthood. Circulation 2013; 127: 905-12.

39. Vaillant GE. Triumphs of Experience. Cambridge, USA:
Harvard University Press; 2012. p. 1-480.

40. Steptoe A, Wardle J, Marmot M.Positive affect and health-related neuroendocrine, cardiovascular, and inflammatory processes. Proc Natl Acad Sci USA 2005; 102: 6508-12.

41. Lawler KA, Younger JW, Piferi RL. A change of heart: cardiovascular correlates of forgiveness in response to interpersonal conflict. J Behav Med 2003; 26: 373-93.

42. Gerin W, Pieper C, Levy R, Pickering TG. Social support in social interaction: a moderator of cardiovascular reactivity. Psychosom Med 1992: 54: 324-36.

43. Ikeda A, Schwartz J, Peters JL, Fang S, Spiro A 3rd, Sparow $\mathrm{D}$, et al. Optimism in relation to inflammation and endothelial dysfunction in older men: the VA Normative Aging Study. Psychosom Med 2011; 73: 664-71.

44. Kelloniemi H, Ek E, Laitinen J. Optimism, dietary habits, body mass index and smoking among young Finnish adults. Appetite 2005; 45: 169-76.

45. Grant N, Wardle J, Steptoe A. The relationship between life satisfaction and health behavior: a cross-cultural analysis of young adults. Int J Behav Med 2009; 16: 25968.

46. Williams GC, Gagne M, Mushlin AI, Deci E. Motivation for behavior change in patients with chest pain. Health Educ 2005; 105: 304-21.

47. Glazer KM, Emery CF, Frid DJ, Banyasz RE. Psychological predictors of adherence and outcomes among patients in cardiac rehabilitation. J Cardiopul Rehab 2002; 22: 42-6.

48. Cotter KA, Lachman ME. No strain, no gain: psychosocial predictors of physical activity across the adult lifespan. J Phys Act Health 2010; 7: 584-94.

49. Giltay EJ, Geleijnse JM, Zitman FG, Buijsse B, Kromhout D. Lifestyle and dietary correlates of dispositional optimism in men: The Zutphen Elderly Study. J Psychosom Res 2007; 63: 483-90.

50. Baruth M, Lee DC, Sui X, Church TS, Marcus BH, Wilcox S, et al. Emotional outlook on life predicts increases in physical activity among initially inactive men. Health Educ Behav, 38: 150-8.

51. Carvajal SC, Wiatrek DE, Evans RI, Knee CR, Nash SG. Psychosocial determinants of the onset and escalation of smoking: Cross-sectional and prospective findings in multiethnic middle school samples. J Adolesc Health 2000; 27: 255-65.

52. Steptoe A, Wright C, Kunz-Ebrecht SR, Iliffe S. Dispositional optimism and health behaviour in communitydwelling older people: Associations with healthy ageing. Brit J Health Psychol 2006; 11: 71-84.

53. Niemiec CP, Ryan RM, Patrick H, Deci EL, Williams GC. The energization of health-behavior change: Examining 
Recursos psicosociales positivos y salud cardiovascular - M. P. Nitsche et al

the associations among autonomous self-regulation, subjective vitality, depressive symptoms, and tobacco abstinence. J Positive Psychol 2010; 5: 122-38.

54. Di Matteo MR. Social support and patient adherence to medical treatment: a meta-analysis. Health Psychol 2004; 23: 207-18.

55. Ades PA. Cardiac rehabilitation and secondary prevention of coronary heart disease N Engl J Med 2001; 345: 892-902.

56. Seligman ME, Steen TA, Park N, Peterson C. Positive psychology progress: empirical validation of interventions. Am Psychol 2005; 60: 410-21.

57. Peterson JC, Charlson ME, Hoffman Z, Wells MT, Wong SC, Hollenberg JP, et al. A randomized controlled trial of positive-affect induction to promote physical activity after percutaneous coronary intervention. Arch Intern
Med 2012; 172: 329-36.

58. Ogedegbe GO, Boutin-Foster C, Wells MT, Allegrante JP, Isen AM, Jobe JB, et al. A randomized controlled trial of positive-affect intervention and medication adherence in hypertensive African Americans.Arch Intern Med 2012; 172: 322-6.

59. Van Dalem J, Krass I, Aslani P. Interventions promoting adherence to cardiovascular medicines. Int J Clin Pharm 2012; 34: 295-311.

60. Ryan RM, Deci EL. Self-determination theory and the facilitation of intrinsic motivation, social development, and well-being. Am Psychol 2000; 55: 68-78.

61. Ryan RM, Patrick H, Deci EL, Williams GC. Facilitating health behaviour change and its maintenance: interventions based on self-determination theory. Eur Health Psychol 2008; 10: 102-5. 